\title{
A NOTE ON NORMAL MATRICES
}

\author{
Marvin Marcus and Nisar Khan (1)
}

(received October 7,1960 )

Introduction. In 1954 A. J. Hoffman and O. Taussky [1] showed that if $A$ is an $n$-square complex matrix with eigenvalues $\lambda=\left(\lambda_{1}, \ldots, \lambda_{n}\right)$ and $P$ is a permutation matrix for which $\alpha A+\beta A *$ has eigenvalues $\alpha \lambda+\beta P \bar{\lambda}$ for some $\alpha \beta \neq 0$ then $A$ is normal. Here $\bar{\lambda}$ is the conjugate vector of $\lambda$. As a companion result they also proved that if the eigenvalues of $A A$ * are $\lambda_{i}(\bar{P} \lambda)_{i}, i=1, \ldots, n$ then $A$ is normal. (See footnote.)

In this note we obtain similar characterizations of normal matrices. Our main results are contained in the

THEOREM. Let $\alpha$ be a complex number, $0 \neq|\alpha| \neq 1$, and let $\lambda=\left(\lambda_{1}, \ldots, \lambda_{n}\right)$ be the eigenvalues of $A$. If $S$ is a real orthogonal matrix and $\alpha A+A *$ has eigenvalues $S(\alpha \lambda+\bar{\lambda})$ then $A$ is normal. If $U$ is unitary and $A A *$ has eigenvalues $\lambda_{i}\left(\overline{U \lambda}_{i}, i=1, \ldots, n\right.$ then it also follows that $A$ is normal.

We prove the first part of the theorem in a sequence of lemmas.

The second part is very easy and we indicate this at the end of the paper.

Editor's footnote. A* denotes the conjugate complex transpose of A. A is called normal if it commutes with $A *$. By a theorem of Schur and Toeplitz, $A$ is normal if and only if the re exists a unitary matrix $U$ and a diagonal matrix $D$ such that $U * A U=D$. Cf. Linear Algebra and Matrices, by H. W. E. Schwerdtfeger, (Groningen, 1950), p. 204.

${ }^{(1)}$ The work o this author was completed under a Postdoctorate Fellowship of the National Research Council of Canada.

Canad. Math. Bull. vol. 4, no. 1, January 1961 
A lower triangular matrix $L$ satisfies $I_{i j}=0$ for $i \leq j$. An upper triangular matrix is the transpose of a lower triangular matrix. If $X$ is any $n$-square matrix $\|x\|$ will denote the Frobenius norm of $\mathrm{X}$ :

$$
\left(\Sigma_{i, j=1}^{n}\left|x_{i j}\right|^{2}\right)^{\frac{1}{2}}=(\operatorname{tr}(X X *))^{\frac{1}{2}}
$$

LEMMA 1. If $L$ is lowe $r$ triangular and $\alpha, \beta$ a re complex numbers then

$$
\operatorname{tr}\left[(\alpha L+\beta L *)^{2}\right]=2 \alpha \beta\|L\|^{2}
$$

Proof.

$\operatorname{tr}\left[(\alpha L+\beta L *)^{2}\right]=\alpha^{2} \operatorname{tr}\left(L^{2}\right)+2 \alpha \beta \operatorname{tr}(L L *)+\beta^{2} \operatorname{tr}\left(L *^{2}\right)$.

Note that the set of lower triangular matrices is closed under multiplication and hence this last expression becomes $2 \alpha \beta\|L\|^{2}$.

LEMMA 2. If $d=\left(d_{1}, \ldots, d_{n}\right)$ satisfies $\alpha d+\bar{d}=S(\alpha \lambda+\bar{\lambda})$ for $|\alpha| \neq 1$ and $S$ is real orthogonal then

$$
\sum_{k=1}^{n} d_{k}^{2}=\sum_{k=1}^{n} \lambda_{k}^{2}
$$

Proof. Let $\lambda=a+i b, d=x+i y, \alpha=w+i \delta$ where $a, b$, $x, y, w$, and $\delta$ a re real. Then equating real and imaginary parts separately of $\alpha d+\bar{d}=S(\alpha \lambda+\bar{\lambda})$ we have

$$
(w+1) x-\delta y=S[(w+1) a-\delta b]
$$

$$
\delta x+(w-1) y=S[\delta a+(w-1) b]
$$

Denote the $2 n$-square matrix

$$
\left(\begin{array}{cc}
(w+1) I & -\delta I \\
\delta I & (w-1) I
\end{array}\right)
$$

by $F$. Now $\operatorname{det} F=\left(|\alpha|^{2}-1\right)^{n} \neq 0$ and hence from(1) we conclude

$$
x+y=(S+S)(a+b)=S a+S b
$$

where $\dot{t}$ indicates direct sum. Thus 


$$
\begin{aligned}
\sum_{k=1}^{n} d_{k}^{2} & =\sum_{k=1}^{n}\left(x_{k}+i y_{k}\right)^{2} \\
& =(x, x)+2 i(x, y)-(y, y) \\
& =(S a, S a)+2 i(S a, S b)-(S b, S b) \\
& =(a, a)+2 i(a, b)-(b, b) \\
& =\sum_{k=1}^{n}\left(a_{k}+i b_{k}\right)^{2} \\
& =\Sigma_{k=1}^{n} \lambda_{k}^{2} .
\end{aligned}
$$

Here (, ) indicates the usual unitary inner product of two n-tuples.

To proceed to the proof of the first part of the theorem select a unitary matrix $R$ that brings $\alpha A+A^{*}$ to triangular form with zero below the main diagonal. Let $B=R A R^{*}$ and set

$$
B=D+L+V
$$

where $D=\operatorname{diag}\left(d_{1}, \ldots, d_{n}\right)$ and $L$ and $V$ are lower and upper triangular matrices. Then

$$
\alpha B+B *=(\alpha D+\bar{D})+(\alpha L+V *)+(\alpha V+L *)
$$

Let $d=\left(d_{1}, \ldots, d_{n}\right)$ and let $\gamma=\left(\gamma_{1}, \ldots, \gamma_{n}\right)$ be the $n-t u p l e$ of numbers on the main diagonal of $\alpha B+B *$, i.e., the eigenvalues of $\alpha A+A *$. Then

$$
\begin{gathered}
\alpha \mathrm{d}+\overline{\mathrm{d}}=\gamma=S(\alpha \lambda+\bar{\lambda}), \\
\alpha \mathrm{L}+\mathrm{V} *=0,
\end{gathered}
$$

and we conclude that

$$
B=D+L-\bar{\alpha} L * .
$$

By lemma 2,

$$
\sum_{k=1}^{n} d_{k}^{2}=\sum_{k=1}^{n} \lambda_{k}^{2}
$$


and hence

$$
\operatorname{tr}\left(B^{2}\right)=\sum_{k=1}^{n} \lambda_{k}^{2}=\operatorname{tr}\left(D^{2}\right)
$$

But

$$
\begin{aligned}
\operatorname{tr}\left(B^{2}\right) & =\operatorname{tr}\left[\left(D+L-\bar{\alpha} L^{*}\right)^{2}\right] \\
& =\operatorname{tr}\left(D^{2}\right)+2 \operatorname{tr}\left[\left(L-\bar{\alpha} L^{*}\right) D\right]+\operatorname{tr}\left[\left(L-\bar{\alpha} L^{*}\right)^{2}\right]
\end{aligned}
$$

Note that $\operatorname{tr}[(L-\bar{\alpha} L *) D]=0$ and thus

$$
\operatorname{tr}\left[\left(L-\bar{\alpha} L^{*}\right)^{2}\right]=0 \text {. }
$$

By lemma 1

$$
-2 \bar{\alpha}\|L\|^{2}=0
$$

and hence

$$
\mathrm{L}=0 \text {. }
$$

Thus $B=R A R *$ is a diagonal matrix and from this it follows that $A$ is normal.

To see the last part of the theorem note that

$$
\begin{aligned}
0 \leq \operatorname{tr}(\mathrm{AA} *)=(\lambda, U \lambda) & =|(\lambda, U \lambda)| \leq\|\lambda\|\|U \lambda\| \\
& =\|\lambda\|^{2}=\sum_{i=1}^{n}\left|\lambda_{i}\right|^{2} .
\end{aligned}
$$

Thus if $R A R *=\operatorname{diag}\left(\lambda_{1}, \ldots, \lambda_{n}\right)+L$, where $L$ is lower triangular and $R$ is unitary then

$$
\operatorname{tr}\left(A A^{*}\right)=\sum_{i=1}^{n}\left|\lambda_{i}\right|^{2}+\|L\|^{2} \leq \sum_{i=1}^{n}\left|\lambda_{i}\right|^{2}
$$

and $L=0$. This completes the proof. 


\section{REFERENCE}

1. A. J. Hoffman and O. Taussky, A characterization of normal matrices, J. Research, Nat. Bur. Standards $52(1954), 17-19$.

U. S. National Bureau of Standards

Washington, D. C.

and

Muslim University

Aligarh, India

and

University of British Columbia 